\title{
Comparison of dielectric materials for the activation of a macro-scale hinge configuration
}

\author{
C. Jordi ${ }^{a, b^{*}}$, A. Schmidt ${ }^{a, b}$, G. Kovacs ${ }^{a}$, P. Ermanni ${ }^{b}$ \\ ${ }^{a}$ Empa, Swiss Federal Laboratories for Materials Testing and Research, Laboratory for Mechanical \\ Systems Engineering, Ueberlandstr. 129, 8600 Duebendorf, Switzerland \\ ${ }^{b}$ ETH, Swiss Federal Institute of Technology Zurich, Centre of Structure Technologies, Switzerland
}

\begin{abstract}
While much of the research on dielectric elastomer actuators used to concentrate on VHB 4910 as dielectric material, lately many new, specifically developed materials have come into focus. The acrylic VHB has been thoroughly characterized in a macro-scale agonist-antagonist configuration on an active hinge. This was carried out with the aim of using it on an airship, which was activated, undulating body and a fin and thus propelled in a fish-like manner. The concept was proved in flight, but still lifetime and viscosity of the actuators and the time-costing fabrication due to the necessary large pre-stretches of the dielectric membrane caused severe inconveniences. In order to evaluate the usability of other materials for this specific purpose, two other materials, a corrugated silicone with silver electrodes (by PolyPower) and an acrylic with interpenetrating network (IPN) developed by Pei et al. were characterized under similar conditions. The influence of the material on performance and design of the actuators and the conclusions for the use of the materials on the airship (and on applications with similar performance requirements) are presented.
\end{abstract}

Keywords: Dielectric elastomers (DE), Electro-active polymers (EAP), Interpenetrating Polymer Network (IPN), Silicone, Biomimetic airship

\section{INTRODUCTION}

Dielectric elastomers (DE) as transducers have been studied extensively in the past years. As dielectric material VHB 4910 (by 3M) was used widely and it has been thoroughly investigated, mainly in actuator applications [1-3]. This is due to the large active deformation that can be achieved, its commercial availability and overall good activation performance. Our group has developed large planar DE actuators made from VHB 4910 for the application on an airship [4]. The 8 meter model airship was equipped with a tail fin and actuators on either side of the body and fin. By activating them alternately, the airship was propelled with an undulating movement of body and fin - similar to a fish (Fig.1). The acrylic material has some severe disadvantages on the other hand: a large pre-stretch is necessary in order to decrease thickness

*christa.jordi@empa.ch; phone +41-44-823 4772; fax +41 44823 4011; www.empa.ch/airship

Electroactive Polymer Actuators and Devices (EAPAD) 2011, edited by Yoseph Bar-Cohen, Federico Carpi, Proc. of SPIE Vol. 7976, 79761G · (C) 2011 SPIE · CCC code: 0277-786X/11/\$18 - doi: 10.1117/12.880431 
and stiffness and increase break-down strength [5,6], it has a high viscoelasticity, it is adhesive (which may be an advantage or a disadvantage) and the temperature range where it is suitable as dielectric is very limited. During this project, these disadvantages caused considerable additional efforts:

- The actuators had to be internally reinforced with carbon rods and polyamide rope, due to the large contraction in the direction perpendicular to the active. This lead to an increase in fabrication time, more failure and waste during the fabrication, additional weight and a short lifetime of the actuators due to the stress concentrations.

- The forces of the pre-stretch of the actuators had to be absorbed by the hull and finally by the internal structure of the airship and the structure of the tail fin. This lead to additional weight.

- Because the actuators were still a bit adhesive, over time they would sometimes stick to the hull and this caused premature failure of the actuators usually and damage of the hull sometimes.

- $\quad$ The system was constructed for $0.1 \mathrm{~Hz}$ actuation. When the frequency was increased (which might have been favorable for the propulsion), the active strain that we could reach with the actuators and thus the amplitude of the undulation decreased, due to viscoelastic effects.

(a)

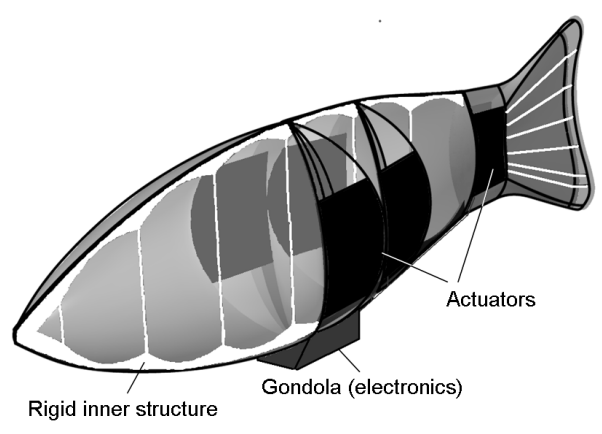

(b)

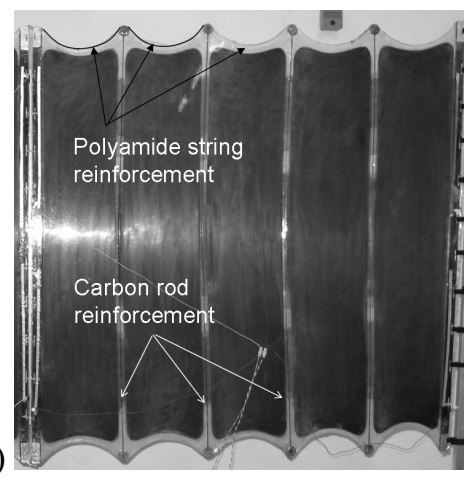

Fig. 1: Configuration of the model airship (a). Actuator design for the airship (b).

The objective of this paper is now to compare two other materials that have come into view more recently, to the VHB 4910. The first material, we will call it IPN (Interpenetrating Polymer Network), was developed by Pei et al. [7-9]. It is a pre-stretched VHB 4910 membrane where the pre-stretch is partially preserved internally by a second, stiffer polymer network under compression. Like pre-stretched VHB, IPN displays a high break-down field, while it does not require an external structure to maintain the pre-stretch. The second material (by PolyPower) consists of two laminated layers of silicone $[10,11]$. The outer surfaces are corrugated in one direction and coated with a thin layer of silver. Since the stiffness of the silver layer is around $10^{5}$ times that of the silicone, this introduces a pronounced structural anisotropy. The film in total is much stiffer in one direction and very compliant in the perpendicular, corrugated direction (Fig.2). In that direction the material can be stretched up to $100 \%$ approximately without fracturing the silver coating. 
(a)

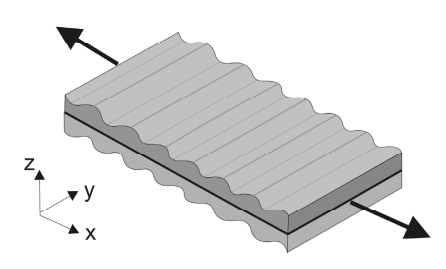

(b)

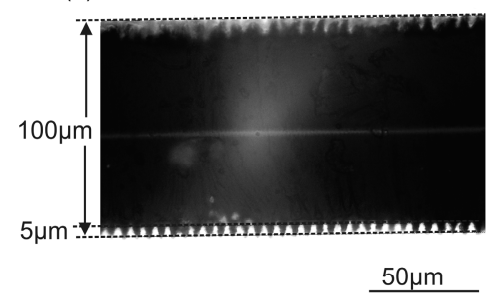

Fig. 2: Corrugated silicone film with silver electrodes (PolyPower DEAP).

For the development and characterization of the large-scale VHB 4910 actuators, an agonist-antagonist hinge configuration had been chosen $[12,13]$. In the hinge we have a similar configuration as on the airship, we can measure necessary performance parameters, such as blocking moment and deflection angle, and the scalability was proofed in systematic comparison. The same hinge will be used to compare the different materials now. The objective is to characterize the passive materials under similar conditions (e.g. strain rates, geometry, etc.), describe the influence of the various material parameters on the design and performance, compare them on a large-scale agonist-antagonist configuration and conclude on their suitability for the application on the airship.

\section{EXPERIMENTAL CHARACTERIZATION}

\subsection{Sample preparation}

For VHB 4910 the optimal pre-stretch for maximum active strain was found to be around $\lambda_{x}=3.5, \lambda_{y}=6$ (with $\lambda=$ strain +1 , $\mathrm{x}$ being the active direction (Fig.2)) [5]. Nevertheless, for the following measurements a pre-stretch of $\lambda x=3$ and $\lambda y=5$ was used. This allows for easier fabrication and longer lifetimes and it is also how the reference hinge was made $[12,13]$. As electrodes for the active tests, a carbon powder (KetjenBlack600) was rubbed onto the membranes.

The IPN is fabricated by pre-stretching VHB 4910 to $400 \%$ equibiaxially onto a frame. It is then sprayed with a monomer and cross-linked in a vacuum oven. Upon releasing the material from this frame, the material contracts to roughly $200 \%$ pre-stretch in the VHB film, which is then the new reference state. The material was used directly from the fabrication frames for simpler processing. From thickness measurements it can be gathered that the pre-stretch relative to the new reference state is approximately $\lambda_{x}=\lambda_{y}=1.4$. The electrode is the same as for the VHB hinges.

The corrugated silicone was tested at $\lambda_{x}=1.4,1.6$ and 1.8 pre-stretch in the active direction (the pre-stretch in the perpendicular direction is always $\lambda_{y}=1$ due to the silver-electrodes that stiffen the material) and the best results were gained with a $40 \%$ pre-stretch [14]. The material is purchased with the silver electrodes, which can be removed with a chlorine solution and a paintbrush where they are not desired.

\subsection{Permittivity}

Permittivity measurements were carried out with an LCR meter (Agilent type $4263 \mathrm{~B}$ ) at $1 \mathrm{kHz}$. Previous measurements were done with rigid copper-electrodes, but did not produce consistent results for the silicone film, since the corrugated 
surface does either have to be squeezed a lot or air will be trapped between the rigid electrodes. Instead, the silver electrode of the film was used directly by removing it with a chlorine solution except for a circular area of $28.5 \mathrm{~mm}$ in the center. To have comparable measurements with VHB 4910 and IPN, sheet-gold electrodes were applied to the adhesive surface with a diameter of $28.5 \mathrm{~mm}$, while the rest of the set-up remained exactly the same (Fig. 3). As verification, undeformed VHB 4910 was measured and a value of 4.75 was gained which agrees well with literature [5]. The permittivity was measured at the pre-stretched state of the material (see Section 2.1). Two to four samples were measured for each material, with a maximum deviation of $5 \%$. The mean values for the pre-stretched materials are 3.9 for VHB 4910, 3.8 for the IPN, and 2.6 for the corrugated silicone.

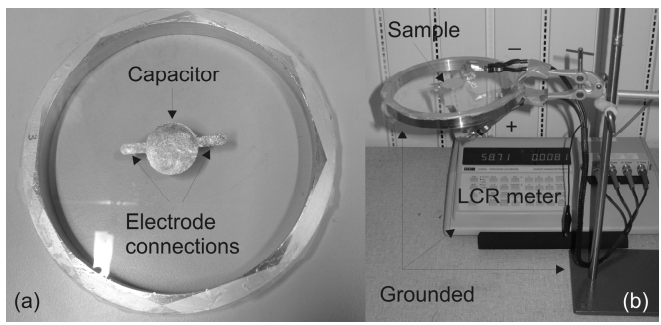

Fig. 3: Sample for permittivity measurements (VHB with sheet-gold electrodes) (a). Test set-up (b). LCR meter, fixations and sample frame are grounded together and the capacity is put to zero before the connections are attached and the sample is measured.

\subsection{Electrical break-down field}

The maximum electric voltage the material can withstand without electric break-down was measured on the prestretched film between rigid brass electrodes of $25 \mathrm{~mm}$ diameter with a radius at the edge of $3.2 \mathrm{~mm}$ in order to prevent border effects. The voltage was increased with steps of $100 \mathrm{~V}$ every second. The thickness was measured with a precision thickness gauge (MT-30 by Heidenhain). The break-down field turned out to be strongly dependent of the stretch state and was therefore investigated over a range of varying stretches. The results are shown in Figure 4. Six samples were measured for VHB 4910. In order to verify the progression as higher stretches, values from literature (Kofod et al. [5]) were added. Eight or more samples were measured for the other materials. From these results an approximation of the break-down field depending on the thickness of the material can be deduced which was used for the performance calculations in Section 3.

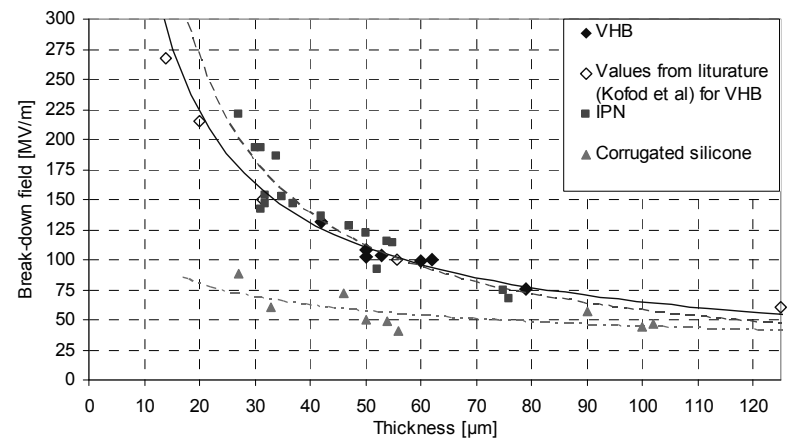

Fig. 4: Break-down field versus thickness (through stretching of the material). Included are results from literature [5] for the verification with VHB at large stretches. 


\subsection{Performance tests on an active hinge configuration}

The hinge for agonist-antagonist activation consists of a rigid structure with a DE actuator on each side $[12,13]$. When activating one side, the opposite actuator contracts due to the pre-stretch and the hinge is deflected. The angle is measured with an angle sensor (type QP-2HC-SW4 by Pewatron AG) while the hinge is fixed vertically on the longer part to reduce inertial effects (Fig. 5 (a)). For the measurement of the blocking moment, the hinge was fixed the other way round and its movement blocked by a force gauge (type HBM S2 by HBM). Only the actuator opposite the force gauge was activated such that scant movement of the hinge is possible (Fig. 5 (b)).
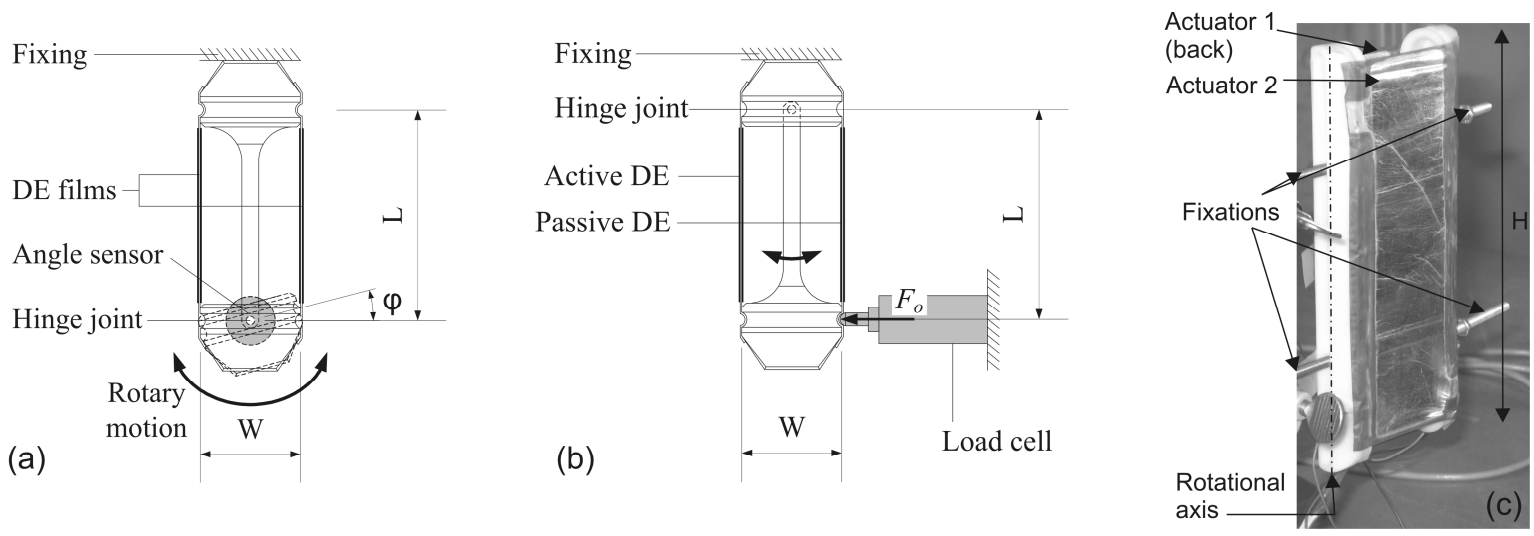

Fig. 5: Hinge design and set-up for deflection angle measurements (a) and blocking force measurements (b).

Hinge with silicone actuators (c).

The maximum deflection angles versus applied activation voltage are shown in Figure 6 (a). The angles at an activation frequency of $0.1 \mathrm{~Hz}(5 \mathrm{~s}$ active per actuator) are shown. The largest angles at a certain activation voltage were clearly reached with VHB 4910, while about half the deflections were obtained with the silicone and the IPN film. Since all of these materials have a different thickness though, the angles were plotted versus the initial electric field (activation voltage divided by thickness in the pre-stretched state, Fig. 6 (b)). It can be seen that we can reach about the same angles with VHB and IPN, but the necessary field is much higher for the IPN. With the silicone, the deflections are higher at lower electrical fields than with IPN, but lower than with VHB. This is due to the lower relative permittivity and slightly higher stiffness of the silicone. To increase the maximum deflection, the break-down strength of the corrugated silicone film would have to be improved. IPN on the other hand has a high break-down strength (although Fig. 4 shows a similar result for IPN and VHB we gather from thickness measurements, that the VHB membrane enclosed in the IPN must be much more pre-stretched and thus have a much higher break-down strength). The material is too stiff though and the needed electrical field is therefore higher. The time dependent behavior is shown in Figure 7 where the maximum deflection angle is depicted over the frequency. The deflection angle that is reached with VHB and IPN decreases with increasing frequency. On the other hand the angle that can be reached with the corrugated silicone remains constant. It even increases slightly at frequencies above $2 \mathrm{~Hz}$, which is presumably due to mass effects. These results clearly show the potential of silicones for applications with higher frequencies. 

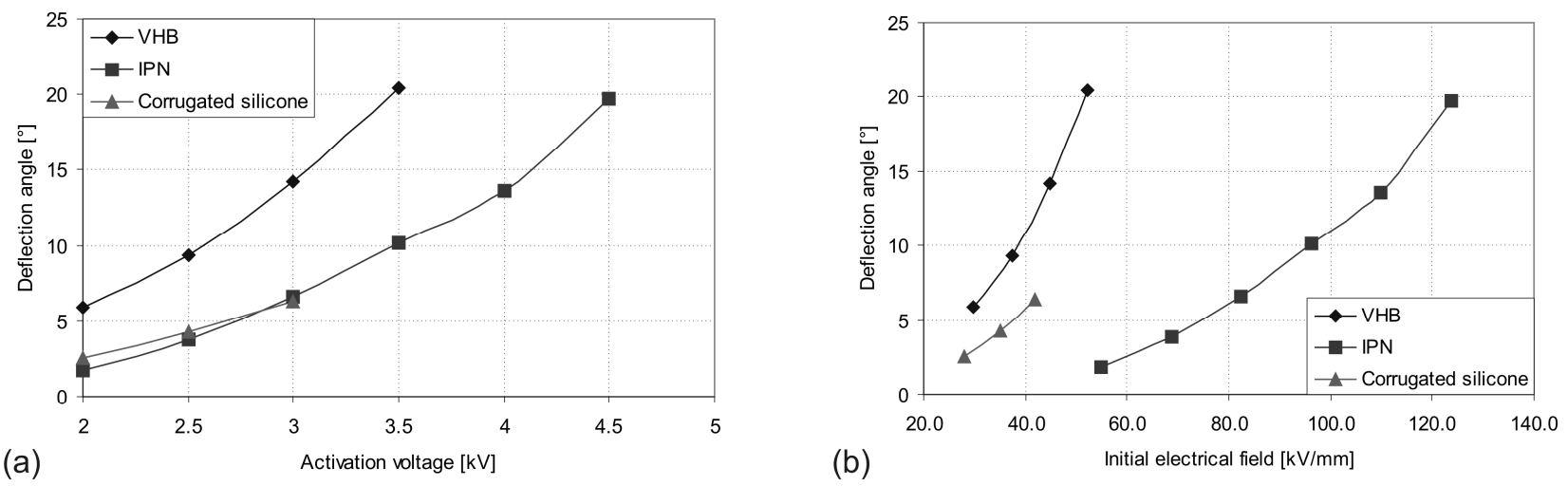

Fig. 6: Deflection angle vs. applied voltage (a) and vs. initial electric field (b). Comparing VHB, IPN and silicone at $0.1 \mathrm{~Hz}$.

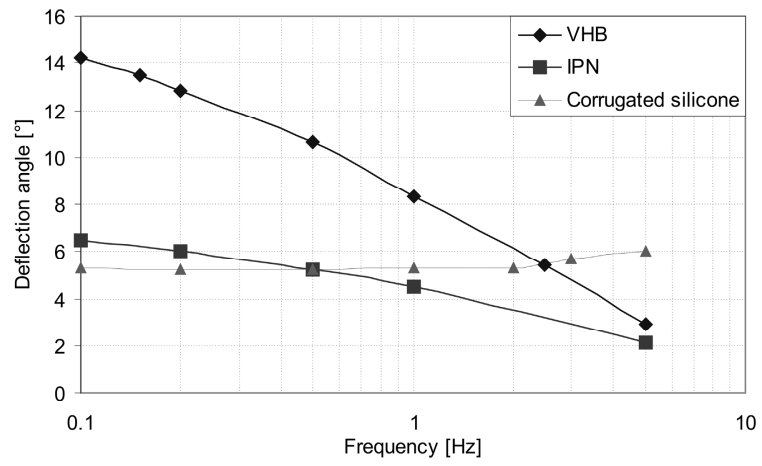

Fig. 7: Deflection angle vs. activation frequency. Comparing VHB, IPN and silicone at $3 \mathrm{kV}$ activation voltage.

The blocking moment is calculated from the measured blocking force $\Delta F$ with $M=\Delta F \cdot W / 2$ and normalized with the height of the hinge $(m=M / H)$ (Fig. $5(\mathrm{~b}))$. Figure 8 shows this normalized blocking moment versus the activation voltage. We can scale the blocking moment linearly with the number of layers of the actuators. From the results we can see that to reach the same blocking moment we can reach with one layer of the VHB approximately two layers of the IPN or three layers of the corrugated silicone film are necessary.

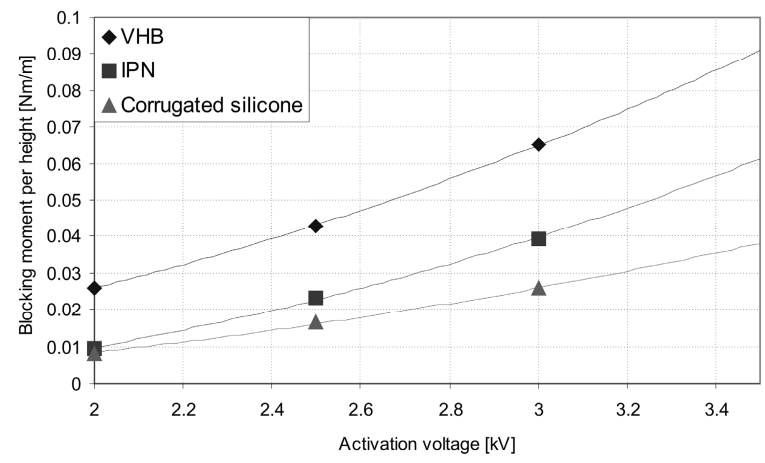

Fig. 8: Blocking force per unit height vs. applied voltage. Comparing VHB, IPN and silicone at $0.1 \mathrm{~Hz}$. 


\section{PERFORMANCE CALCULATIONS}

In addition to the experimental characterization, the theoretical performance of the hinge with each material was calculated. If an electric field is applied across the thickness of an incompressible film, the electrostatic pressure equivalent to the apparent three-dimensional state of stress can be calculated by Equation 1 (with $\varepsilon_{0}$ permittivity of vacuum, $\varepsilon_{r}$ the relative dielectric permittivity of the elastomer, $E$ the electrical break-down field, $V$ the activation voltage, $t$ the thickness of the deformed membrane and $T$ the thickness of the undeformed membrane as it is purchased). The permittivity $\varepsilon_{r}$ and the maximum electric field the material can withstand without electric breakdown $E_{b d}$ were shown to depend on the state of deformation in Section 2.3 and in [5]. While the dependence of $E_{b d}$ on deformation is very pronounced (Fig. 4), $\varepsilon_{r}$ only changes a few percent within the range of active deformation $[5,15]$. We will thus subsequently assume a constant value of permittivity throughout the activation. This justifies the use of Equation 1 instead of a recently proposed electrostrictive model [16].

$p_{e l}=\varepsilon_{r} \cdot \varepsilon_{0} \cdot E^{2}$ with $\quad E=\frac{V}{t} \quad$ with $\quad t=T \cdot \lambda_{z}$

Since all materials investigated reveal a non-linear mechanical behavior, hyperelastic material models of Yeoh (VHB, corrugated silicone, Eq. 2) and Ogden (IPN, Eq. 3) are used instead to calculate active deformations. The parameters for the material models are listed in Table 1. For the VHB 4910 the parameters of Wissler et al. [17] were used and for the corrugated silicone and IPN the parameters were established by Schmidt et al. [18,19].

$$
\begin{aligned}
& W_{\text {Yeoh }}=C_{10}\left(I_{1}-3\right)+C_{20}\left(I_{1}-3\right)^{2}+C_{30}\left(I_{1}-3\right)^{3} \text { with } I_{1}=\lambda_{x}^{2}+\lambda_{y}^{2}+\lambda_{z}^{2} \\
& W_{\text {Ogden }}=\sum_{i} \frac{2 \mu_{i}}{\alpha_{i}^{2}}\left(\lambda_{x}^{\alpha_{i}}+\lambda_{y}^{\alpha_{i}}+\lambda_{z}^{\alpha_{i}}-3\right)
\end{aligned}
$$

The stress in the material in the active direction can then be calculated as a function of the stretching factors $\lambda$ with:

$$
\sigma_{x}=\lambda_{x} \frac{\partial W}{\partial \lambda_{x}}-\lambda_{z} \frac{\partial W}{\partial \lambda_{z}}
$$

Table 1: Parameter for the hyperelastic material models.

\begin{tabular}{lllllll}
\hline Yeoh parameter & $\mathrm{C}_{10}[\mathrm{MPa}]$ & $\mathrm{C}_{20}[\mathrm{MPa}]$ & $\mathrm{C}_{30}[\mathrm{MPa}]$ & & & \\
\hline VHB 4910 [17] & 0.05374 & -0.0004857 & $3.809 \mathrm{e}-6$ & & & \\
\hline Corrugated silicone $^{1}[19]$ & 0.08119 & -0.00539 & 0.00169 & & & \\
\hline Ogden parameter & $\mu_{1}[\mathrm{MPa}]$ & $\alpha_{1}[-]$ & $\mu_{2}[\mathrm{MPa}]$ & $\alpha_{2}[-]$ & $\mu_{3}[\mathrm{MPa}]$ & $\alpha_{3}[-]$ \\
\hline IPN $^{2}[18]$ & 0.386 & 0.206 & 0.119 & 7.182 & -0.066 & 6.397 \\
\hline $\begin{array}{l}{ }^{1} \text { with an initial thickness of } 100 \mu \mathrm{m} \\
{ }^{2} \text { with an initial thickness of } 67 \mu \mathrm{m}\end{array}$ & & & & & & \\
\end{tabular}


The boundary conditions were chosen as for the hinge. The stretch in the perpendicular direction is assumed to be constant $\left(\lambda_{y}=\right.$ const $)$, and the material is modeled incompressible $\left(\lambda_{x} \cdot \lambda_{y} \cdot \lambda_{z}=1\right)$. With $\lambda_{y}$ and $\varepsilon_{r}$ thus being known constants and $\lambda_{z}, E_{b d}$ and $\sigma_{x}$ as functions of $\lambda_{x}$, the equilibrium of forces (Eq. 5) in the deflected hinge can then be solved for the stretchstate of the expanded (active) actuator $\left(\lambda_{a}\right)$ and the active strain and deflection angle can be calculated. $\lambda_{c}$ is the stretch in the contracted actuator, which is equal to two times the pre-stretch $\left(\lambda_{p s}\right)$ minus the stretch in the active actuator.

$$
\sigma\left(\lambda_{c}\right) \cdot d\left(\lambda_{c}\right)=\left(\sigma\left(\lambda_{a}\right)-p_{e l}\left(\lambda_{a}\right)\right) \cdot d\left(\lambda_{a}\right) \quad \text { with } \quad \lambda_{c}=2 \lambda_{p s}-\lambda_{a}
$$

Figure 9 shows the results of the calculated deflection angles. They are compared to the measured results (Section 2.4, Fig. 6). We can see that the predictions are good concerning the angle for VHB and the corrugated silicone. For the IPN material the predictions are not as good, which is due to the fact, that every IPN membrane has a slightly different stiffness based on the manual fabrication process and there are variations even within one membrane [18]. There is also a variance in the fabrication of the hinge system, which leads to an error in the reported measured angles. In general the observations reported in Section 2.4 are confirmed.

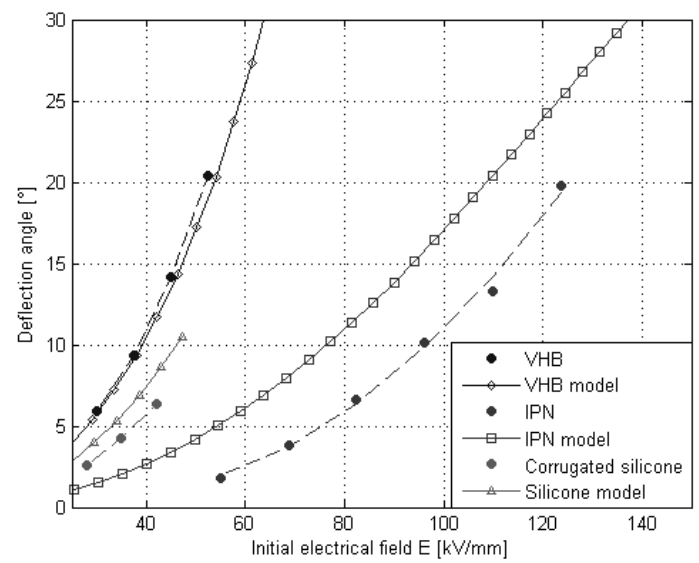

Fig. 9: Comparing the calculated deflection angles with the measurement results.

\section{CONCLUSIONS}

VHB 4910 has long been the most used dielectric for actuators and is therefore well known and characterized. Its exceptional performance was proofed e.g. by activating the hull and tail fin of a fish-like biomimetic airship, but showed some critical disadvantages nevertheless. In the present study, it was compared to two promising materials: IPN from VHB 4910 (developed by Pei et al.) and a corrugated silicone with silver electrodes (by PolyPower). In order to have comparable values, permittivity and break-down field measurements were repeated. The materials were then built into actuators and the performance investigated in an agonist-antagonist hinge configuration that has been used before to predict the behaviour of VHB actuators on the airship. Additionally, calculations were done to the performance that can theoretically be reached. From the theoretical and experimental results we can conclude that the largest deflection angles and blocking force can still be achieved with VHB 4910 among these three materials. On the other hand, the disadvantages that were listed in the introduction can mostly be overcome by the new materials: 
- With IPN the internal reinforcements of the actuators would still be necessary, but fewer. With the same amount of reinforcements, the stress peaks would be much less critical nevertheless. With the corrugated silicone the internal reinforcements would become unnecessary. This would lead to shorter production times and longer lifetimes.

- The forces of the pre-stretch of the actuators are lower in both cases (see also isotonic tests and uniaxial tension test in [19]). This allows for a lighter inner structure of the airship or for a more stable system.

- Actuators made from IPN are still quite adhesive and the same damage might occur. The PolyPower silicone on the other hand is not adhesive at all. This leads to less failure and it may even make the contractions within the hull (Fig.1) unnecessary (if the friction between hull and actuator does not diminish the active strain).

- The silicone shows very little viscoelastic behavior and thus the deflection angles remain almost constant with changing frequency (Fig. 7). Also with IPN actuators the angle decreases less with increasing frequency than with the VHB. This is important because we can then adjust the undulating frequency independent of the undulating amplitude, as the fish does in a certain range.

An airship with IPN actuators could thus reach the same deflection angles with actuators that would be the same length (Fig. 6 (a)). $4.5 \mathrm{kV}$ instead of around $3.5 \mathrm{kV}$ would be necessary though and thus different high voltage sources and transistors to switch the high voltage would be needed, which might lead to additional weight. The actuators would have consist of twice the number of layers (four layers on the body actuators and six layers for the tail fin actuators) or be larger in height to reach the same blocking moment (Fig. 8). This is not necessarily a manufacturing problem, but again leads to additional weight. The actuators may still be optimized by relaxing them after the material fabrication process and choosing an optimal anisotropic pre-stretch, as it is done with VHB.

To propel the airship with actuators made from the corrugated silicone they would have to be twice as long as the existing VHB actuators and three times the number of layers. This leads to a considerable additional weight, some of which may be compensated if the contractions in the hull are not needed (gain in volume and thus lift). It is possible that the silver electrodes make this material much more helium tight than the acrylics and a fully integrated solution where the actuators act as hull at the same time may be a good solution.

\section{ACKNOWLEDGEMENT}

This work is funded by the board of directors of Empa. Giovanni Terrasi is acknowledged for his effort in acquiring funding and creating a good research atmosphere. Many thanks go to Marco Lüchinger who helped in the laboratory and with data processing. The support in practical work by Angelo Scioscia, Martin Molberg and Marcel Rees is greatly acknowledged.

\section{REFERENCES}

[1] Bar-Cohen, Y. [Electroactive Polymer (EAP) actuators as artificial muscles: reality, potential and challenges], SPIE press, Bellingham, Washington (2004). 
[2] Carpi, F., De Rossi, D., Kornbluh, R., Pelrine, R., Sommer-Larsen, P., [Dielectric elastomers as electromechanical transducers], Elsevier, Amsterdam (2008).

[3] Brochu, P., Pei, Q., “Advances in Dielectric Elastomers for Actuators and Artificial Muscles”, Macromol. Rapid Commun., 31, 10-36 (2010).

[4] Jordi, C., Michel, S., Fink, E., "Fish-like propulsion of an airship with planar membrane dielectric elastomer actuators”, Bioinsp. Biomim., 5, 026007 (2010).

[5] Kofod, G, Sommer-Larsen, P., Kornbluh, R., Pelrine, R., "Actuation Response of Polyacrylate Dielectric Elastomers", Journal of Intelligent Material Systems and Structures, 14, 787-793 (2003).

[6] Kofod, G., "The static actuation of dielectric elastomer actuators: how does pre-stretch improve actuation?", Journal of Applied Physics D: Applied Physics, 41, 215405 (2008).

[7] Ha, S.M., Wissler, M., Pelrine, R., Stanford, S., Kovacs, G., Pei, Q., "Characterization of electroelastomers based on interpenetrating polymer networks", SPIE proc. EAPAD, 6524, 524087 (2007).

[8] Ha, S.M., Yuan, W, Pei, Q., Pelrine, R., Stanford. S., "Interpenetrating networks of elastomers exhibiting 300\% electrically-induced area strain", Smart Mater. Struct., 16, 280-287 (2007).

[9] Ha, S.M., Yuan, W, Pei, Q., Pelrine, R., Stanford. S., "New high-performance electroelastomer based on interpenetrating polymer networks", SPIE proc. EAPAD, 6168, 16808 (2006).

[10] Sommer-Larsen, P., Hansen, K., Benslimane, M., "Multilayer actuator and sensor sheets with smart compliant electrodes", Advances in Science and Technology, 61, 169-179 (2008).

[11] Benslimane, M.Y., Kiil, H.E., Tryson, M.J. "Electro-mechanical properties of novel large strain PolyPower film and laminate components for DEAP actuator and sensor applications", Proc. of SPIE, 7642, 764231

[12] Jordi, C., Michel, S., Kovacs, G., Ermanni, P., "Scaling of planar dielectric elastomer actuators in an agonistantagonist configuration", Sensors and Actuators A: Physical, 161, 182-190 (2010).

[13] Lochmatter, P., "Design and characterization of an active hinge segment based on soft dielectric EAPs," Sens. Actuators A: Phys., 141, 577-587 (2008).

[14] Jordi, C., Schmidt, A., Kovacs, G., Ermanni, P., "Performance evaluation of cutting-edge dielectric elastomers for large-scale actuator applications", submitted.

[15] Wissler, M., Mazza, E., "Electromechanical coupling in dielectric elastomer actuators", Sens. Actuators A: Phys., 138, 384-393 (2007).

[16] Zhao, X., Suo, Z., "Electrostriction in elastic dielectrics undergoing large deformation", Journal of Applied Physics, 104, 123530 (2008).

[17] Wissler, M., "Modeling Dielectric Elastomer Actuators", PhD thesis, Swiss Federal Institute of Technology Zurich (Switzerland) (2007). 
[18] Schmidt, A., Bergamini, A., Kovacs, G., Mazza, E., "Multiaxial mechanical characterization of Interpenetrating Polymer Network Reinforced Acrylic Elastomer", Experimental mechanics” accepted (2011).

[19] Schmidt, A., Jordi, C., Mazza, E., "Electro-mechanical modeling of dielectric elastomer transducers with microstructured electrodes", SPIE proc. EAPAD, accepted (2011). 